\title{
Confinement-induced order of tethered alkyl chains at the water/vapor interface
}

\author{
M. Fukuto, ${ }^{1}$ R. K. Heilmann,,${ }^{1} *$ P. S. Pershan, ${ }^{1}$ S. M. Yu, ${ }^{2}$ C. M. Soto, ${ }^{3}$ and D. A. Tirrell ${ }^{4}$ \\ ${ }^{1}$ Department of Physics and Division of Engineering and Applied Sciences, Harvard University, Cambridge, Massachusetts 02138 \\ ${ }^{2}$ Department of Chemistry, University of Wisconsin, Madison, Wisconsin 53706 \\ ${ }^{3}$ Polymer Science and Engineering Department, University of Massachusetts, Amherst, Massachusetts 01003 \\ ${ }^{4}$ Division of Chemistry and Chemical Engineering, California Institute of Technology, Pasadena, California 91125
}

(Received 9 November 2001; published 23 July 2002)

\begin{abstract}
Packing of tethered alkyl chains in Langmuir monolayers of a hairy-rod polypeptide poly $[\gamma-4-(n-$ hexadecyloxy)benzyl $\alpha, L$-glutamate] on water has been studied by x-ray scattering measurements at room temperature. The rods lie parallel to the surface while the alkyl side chains segregate toward the vapor. Results indicate that the herringbone order of the alkyl chains is established initially by one-dimensionally confined chains between aligned rods and grows laterally with compression.
\end{abstract}

DOI: 10.1103/PhysRevE.66.010601

PACS number(s): 68.18.-g, 61.66.Hq, 61.10.Eq, 61.10.Kw

The manner in which linear objects such as simple alkyl chains $\left[-\left(\mathrm{CH}_{2}\right)_{n}-\right]$ and polymers order has been of interest for the past half century $[1,2]$. Chain order affects the basic physical chemistry of lipids [3] and the function of biological membranes [4-8]. Properties of alkyl chains also influence phenomena such as friction and adhesion [9] and the phases of liquid crystals $[10,11]$ and Langmuir monolayers (LM) $[12,13]$. It is well known that in the absence of constraining effects, such as might arise from the tethering of one end of the chain $[14,15]$ or competing effects within the internal structure of block copolymers [16], the local order of alkyl chains is relatively insensitive to the length of the chain [17]. The tendency of alkyl chains to pack similarly in diverse systems is rather robust [13].

Details of chain packing, however, are affected by subtle differences in constraints on chain ends. For example, most LM on water spontaneously form two-dimensional (2D) crystalline phases with quasi-long-range positional order [12]. In contrast, the positional order for monolayers of single-chain thiols on the surface of liquid $\mathrm{Hg}$ is only short range [18]. This variation is attributed to differences in the strength of the chain-chain interaction relative to that between the subphase and the chain end. Similarly, selfassembled monolayers (SAM) of thiols on $\mathrm{Au}(111)$ form single crystals with long-range order $[19,20]$, while SAM of thiols on $\mathrm{Ag}(111)$ [21] and siloxanes on $\mathrm{SiO}_{2}$ [22] exhibit only short-range order.

Different phenomena are observed in the packing of polymer chains longer than some persistence length $l_{p}$ [2]. Free polymers in good solvents can coil to form spheres with a radius of gyration $R_{g} \sim l_{p} N^{1 / 3}$. By contrast, polymers tethered to substrates will not have sufficient room to pack as spheres if the mean distance between tethering sites is less than $R_{g}$, resulting in partial unwinding of the chains to form polymer brushes $(\mathrm{PB})$ [9]. In most studies on $\mathrm{PB}$, the ratio of tethering density to $R_{g}$ is controlled by varying the solvent, which in turn affects $R_{g}$. There have been relatively few

\footnotetext{
* Present address: Space Nanotechnology Laboratory, Center for Space Research, Massachusetts Institute of Technology, Cambridge, MA 02139.
}

studies where tethering is to liquid surfaces, for which LM techniques can be used to obtain a direct measure of the effect of $2 \mathrm{D}$ compressive stress on chain packing. In the experiments by Factor $e t$ al. the packing density was varied by adding or removing molecules from a LM of diblock copolymers on the surface of various solvents [23]. Prinz, Muller, and Maaloum carried out similar studies on polyelectrolyte brushes by physically compressing the LM [24].

We present an x-ray scattering study of the packing of constrained alkyl chains in which the tethering is to rods that form a LM. The chains are the free ends of the side chains $\left[-\left(\mathrm{CH}_{2}\right)_{2}-\mathrm{COO}-\mathrm{CH}_{2}-\mathrm{C}_{6} \mathrm{H}_{4}-\mathrm{O}-\left(\mathrm{CH}_{2}\right)_{15} \mathrm{CH}_{3}\right]$ attached to the rigid cylindrical core of a helical polypeptide. The molecule poly $[\gamma-4-(n$-hexadecyloxy)benzyl $\alpha, L$-glutamate] (C16-O-PBLG) is a "hairy rod" in which hexadecyloxy chains (- $-\mathrm{O}-\mathrm{C} 16$; one chain per monomer) extend out from the $\alpha$-helix PBLG core. C16-O-PBLG forms a LM on water in which the rods are oriented parallel to the surface and locally align parallel to neighboring rods within the LM plane. The hydrophobicity of alkyl chains causes them to segregate toward the film/vapor side of the LM, leaving PBLG cores near the water/film interface. We find that despite the highly complex structure of the molecule, the local packing of $-\mathrm{O}-\mathrm{C} 16$ chains at high lateral pressures displays the herringbone (HB) order that is otherwise only known for densely packed alkyl chains. We show evidence that the HB order is first established in the $1 \mathrm{D}$ regions between aligned rods and grows laterally with compression.

Synthesis of C16-O-PBLG has been described elsewhere [25]. The two samples used were polydisperse "PD325" [MW 149000 (vis); DP 325 (vis), PDI 1.32] and monodisperse "MD76" [MW 34 900; DP 76]. Based on the pitch of $L_{1}=1.5 \AA$ /monomer along the $\alpha$-helix axis, the length of the molecules are $L \sim 500 \AA$ for PD325 and $L=115 \AA$ for MD76. The main structural features observed are the same for the two samples.

The Langmuir trough was described previously [26]. A LM was spread on pure water at a specific area $A$ $>40 \AA^{2} /$ monomer from a chloroform solution. Surface pressure $\Pi$ was monitored by a Wilhelmy plate. X-ray measurements were made on the Harvard/BNL liquid spectrometer [26] at Beamline X22B of the National Synchrotron Light 
Source $(\lambda=1.55 \AA)$. For $\mathrm{x}$ rays striking the film at angle $\alpha$ to the surface and scattered at angles $\beta$ to the surface and $2 \theta$ to the plane of incidence, the wave-vector transfer $\mathbf{q}$ has the surface-normal component $q_{z}=(2 \pi / \lambda)[\sin (\alpha)+\sin (\beta)]$ and the in-plane component $q_{x y}=(2 \pi / \lambda)\left[\cos ^{2}(\alpha)+\cos ^{2}(\beta)\right.$ $-2 \cos (\alpha) \cos (\beta) \cos (2 \theta)]^{1 / 2}$. Angular acceptance of the NaI scintillation detector was controlled by two sets of crossed slits, one closer to the sample cell and the other immediately before the detector. For reflectivity (XR), the fraction $R$ of the incident intensity detected at $\beta=\alpha$ and $2 \theta=0$ (or $q_{x y}$ $=0)$ is measured as a function of $q_{z}=(4 \pi / \lambda) \sin (\alpha)$. The data reported here is the difference between the signal at $2 \theta=0$ and the background measured at $2 \theta$ offsets of $\pm 0.25^{\circ}$. For $q_{z} / q_{c} \geqslant 4-5$, where $q_{c}=(4 \pi / \lambda) \sin \left(\alpha_{c}\right) \quad\left(=0.0218 \AA^{-1}\right.$ or $\alpha_{c}=0.154^{\circ}$ for water) is the critical wave vector for total reflection, XR is described by the formula [27]

$$
R\left(q_{z}\right) / R_{F}\left(q_{z}\right)=\left|\rho_{\infty}^{-1} \int d z[d\langle\rho(z)\rangle / d z] \exp \left(i q_{z} z\right)\right|^{2},
$$

where $R_{F}$ represents the Fresnel reflectivity of an ideally flat and sharp interface between the subphase $\left(\rho=\rho_{\infty}\right)$ and the vapor $(\rho=0)$. The average electron density profile $\langle\rho(z)\rangle$ along the surface normal can be extracted by constructing a model and fitting the calculated $R / R_{F}$ to the data. For grazing incidence diffraction (GID), the incident angle was fixed at $\alpha=0.12^{\circ}\left(<\alpha_{c}\right)$ and intensities scattered away from the incidence plane were measured as a function of $q_{x y}$ with a typical in-plane FWHM (full width at half maximum) resolution of $\delta q_{x y} \sim 0.026 \AA^{-1}$ and an out-of-plane detector acceptance of $\Delta q_{z}=0.11 \AA^{-1}$.

Measured $\Pi-A$ isotherms and XR results are summarized in Fig. 1. A typical GID scan at low $q_{x y}$ is shown in Fig. 2(a), and the extracted interhelix $d$ spacing between aligned $\alpha$ helices are plotted in Fig. 2(b). The ratio $A / L_{1}$ [solid lines in Fig. 2(b)] represents the $d$ spacing expected of close-packed horizontally oriented rods. For comparison, Figs. 1 and 2(b) include the results for the LM of $\alpha$-helical PBLG [28], which lacks the $-\mathrm{O}-\mathrm{C} 16$ group and collapses at $\Pi_{1}$ $\sim 9 \mathrm{dyn} / \mathrm{cm}$. The profiles $\langle\rho(z)\rangle$ for the PBLG LM on water [dashed curves in Fig. 1(d)] can be described by a one-box model with Gaussian roughnesses and are consistent with a single surface layer whose thickness $l_{\mathrm{PBLG}}$ is comparable to the diameter $D_{\mathrm{PBLG}} \sim 13 \AA$ of PBLG. The observed lateral $d$ spacing [diamonds in Fig. 2(b)] is also comparable to $D_{\text {PBLG }}$ and follows the expected $d=A / L_{1}$ behavior for $\Pi<\Pi_{1}$. From this we conclude that the PBLG rods are oriented along the surface and align parallel to their neighbors with an area $A_{\mathrm{PBLG}}=L_{1} D_{\mathrm{PBLG}}=19.5 \AA^{2} /$ monomer.

Similarly, the rods in the C16-O-PBLG LM lie horizontally and align locally. The effect of the $-\mathrm{O}-\mathrm{C} 16$ side chains is to induce a nonuniform profile $\langle\rho(z)\rangle$ across the water/C16-O-PBLG/vapor interface that requires a two-box model [solid lines in Fig. 1(d)]. According to analyses to be described elsewhere [29], the profile parameters $\phi_{\text {core }}$, $\phi_{\text {chain }}, l_{\text {core }}$, and $l_{\text {chain }}$, defined by the extremum points in $\langle\rho(z)\rangle$ and $d\langle\rho(z)\rangle / d z$, are consistent with segregation of the LM into a lower sublayer dominated by the PBLG core and an upper sublayer occupied by $-\mathrm{O}-\mathrm{C} 16$ chains [Fig.
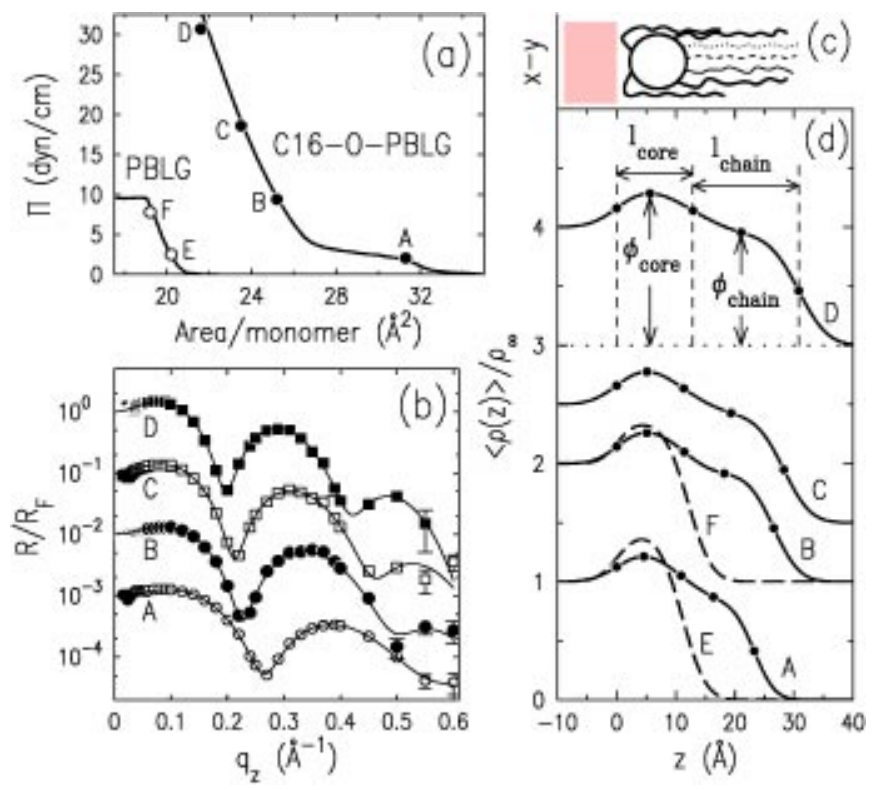

FIG. 1. (a) П- $A$ isotherms for C16-O-PBLG and PBLG films at $22^{\circ} \mathrm{C}$. (b) Data (symbols) and fits (-) for normalized reflectivity $R / R_{F}$ from C16-O-PBLG monolayers (PD325, $22.5^{\circ} \mathrm{C}$ ) at points $A$, $B, C$, and $D$ in (a). (c) An illustration (end-on view) of a hairy rod on water. (d) Best-fit average electron density profiles $\langle\rho(z)\rangle / \rho_{\infty}$ for C16-O-PBLG ( - ) and PBLG $(---)$ monolayers at $A-F$ in (a). Parameters $l_{\text {core }}, l_{\text {chain }}, \phi_{\text {core }}$, and $\phi_{\text {chain }}$ are illustrated $(\bullet)$. Curves in (b) and (d) are shifted for clarity.

$1(\mathrm{c})]$. For example, $l_{\text {core }}$ is comparable to $l_{\mathrm{PBLG}}$, and $\phi_{\text {chain }}$ is bracketed by the values for liquid $(\phi \sim 0.80)$ and solid $(\phi$ $=0.95-1.0)$ phases of alkane chains $[26,30]$. The two sublayers account for all of the electrons in the C16-O-PBLG molecule; the number of electrons per unit area $\rho_{s}$ $=(255$ electrons $/$ monomer $) / A$ expected for C16-O-PBLG and the experimental value $\rho_{\infty}\left(\phi_{\text {core }} l_{\text {core }}+\phi_{\text {chain }} l_{\text {chain }}\right)$ agree within $\sim 10 \%$ of each other for all $\Pi$. These results suggest that the $-\mathrm{O}-\mathrm{C} 16$ chains stabilize the $\mathrm{LM}$ at high $\Pi$.

The observed interhelix $d$ spacing for C16-O-PBLG is larger than that for PBLG by $\Delta d \sim 5 \AA$ [Fig. 2(b)], which is comparable to typical nearest-neighbor $(\mathrm{NN})$ distances found
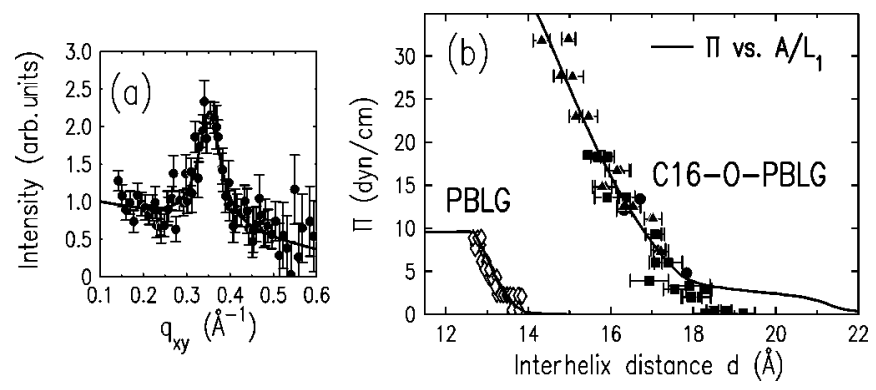

FIG. 2. (a) A GID scan at low $q_{x y}\left(\left\langle q_{z}\right\rangle=0.05 \AA^{-1}\right)$ from a C16-O-PBLG monolayer and a fit $(-)$ based on a Lorentzian with constant and linear background terms. (b) $\Pi$ vs interhelix spacing $d=2 \pi / q_{x y}^{\text {Peak }}$. Filled symbols are from C16-O-PBLG (PD325, $22.5^{\circ} \mathrm{C}$ ) and open from PBLG. Each solid curve is obtained by dividing the area $A$ in the isotherm by the $\alpha$-helix pitch $L_{1}$ $=1.5 \AA$ /monomer. 

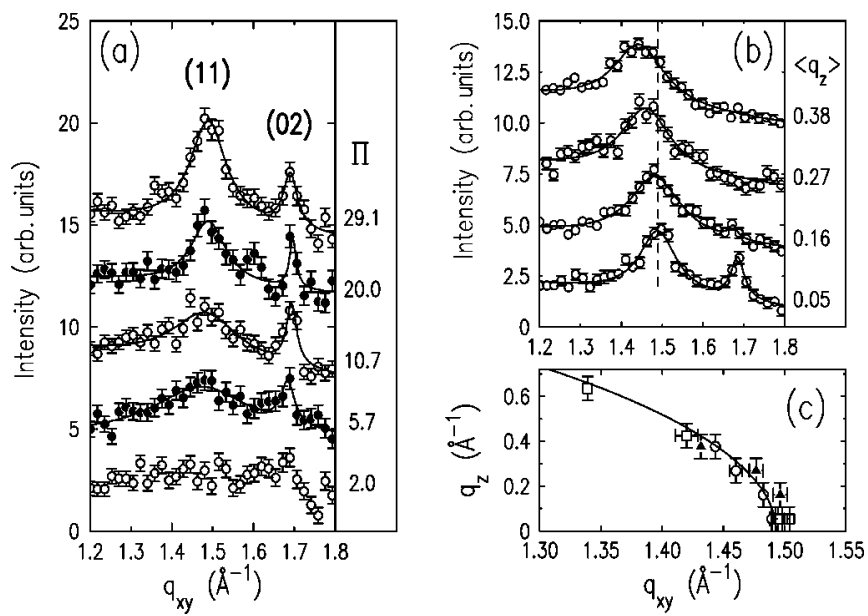

FIG. 3. (a) GID scans at high $q_{x y}\left(\left\langle q_{z}\right\rangle=0.05 \AA^{-1}\right)$ from C16O-PBLG monolayers (MD76, $30.9{ }^{\circ} \mathrm{C}$ ) at various $\Pi$. (b) Scans at various $\left\langle q_{z}\right\rangle$ above the surface (MD76, $\left.22.5^{\circ} \mathrm{C}, \Pi=13.9 \mathrm{dyn} / \mathrm{cm}\right)$. Curves in (a) and (b) are shifted for clarity. (c) Positions of the (11) peak in reciprocal space: MD76 at $13.9 \mathrm{dyn} / \mathrm{cm}(\mathrm{O})$ and 24.0 dyn/cm ( $\square$ ) and PD325 at $31.3 \mathrm{dyn} / \mathrm{cm}(\boldsymbol{\Delta})$, all at $22.5^{\circ} \mathrm{C}$. The vertical error bars represent the detector acceptance $\Delta q_{z}$ $=0.11 \AA^{-1}$. The solid curve is equal to $q=1.493 \AA^{-1}$, where $q^{2}$ $=q_{x y}^{2}+q_{z}^{2}$.

in ordered phases of alkyl chains [12,30]. Given the hydrophobicity of the side chains this extra spacing most certainly arises from chains that are tethered on the water side of the helix backbone and extend toward the vapor. This is supported by the fact that $\phi_{\text {core }}$ is slightly lower than the maximum in the PBLG profile [Fig. 1(d)].

High- $q_{x y}$ GID results summarized in Fig. 3 illustrate chain ordering effects. The positions of peaks (11) and (02) observed at high $\Pi(\gtrsim 5 \mathrm{dyn} / \mathrm{cm})$ are consistent with the $\mathrm{HB}$ arrangement of alkyl chains, with an orthorhombic unit cell (two chains/cell) of dimensions $a_{1} \times a_{2}=5.0 \times 7.5 \AA$ in the plane perpendicular to chain axes $[12,13,17,31]$. The model illustrated in Fig. 4 for the development of the HB order is based on the following observations.

First, the packing order of $-\mathrm{O}-\mathrm{C} 16$ chains is a local effect in that the magnitude of the chain tilt relative to the surface normal is not uniform over the surface. Figure 3(b)3(c) show that at fixed $\Pi$, the (11) peak shifts with $q_{z}$ along an arc $q=\left[q_{x y}^{2}+q_{z}^{2}\right]^{1 / 2} \sim 1.5 \AA^{-1}$, while the (02) is only visible at $q_{z} \sim 0$. This signifies tilts of HB-packed chains toward NN (i.e., toward [10]) with a distribution in tilt angles $\theta$ that vary from $\theta=0^{\circ}$ (untilted) to $\gtrsim 30^{\circ}$, even at high $\Pi$. In view of the tethering of the $-\mathrm{O}-\mathrm{C} 16$ chains the lack of a unique tilt angle is not surprising.

Second, the correlations of the HB order are anisotropic with respect to the alignment of PBLG cores. This can be seen from the fact that the (02) peak is noticeably narrower than the (11) peak [Fig. 3(a)]. Defining $\xi_{h k} \equiv 2 /\left[\Delta q_{x y}(h k)\right.$ $\left.-\delta q_{x y}\right]$ with $\Delta q_{x y}(h k)$ representing the FWHM of the $(h k)$ peak, we estimate the lateral correlation length $\zeta_{01}$ along the [01] axis (i.e., perpendicular to the tilt direction) to be $\zeta_{01}$ $=\xi_{02} \gtrsim 100 \AA$, which is roughly of the same order of magnitude as the lengths $L$ of typical C16-O-PBLG rods. At high

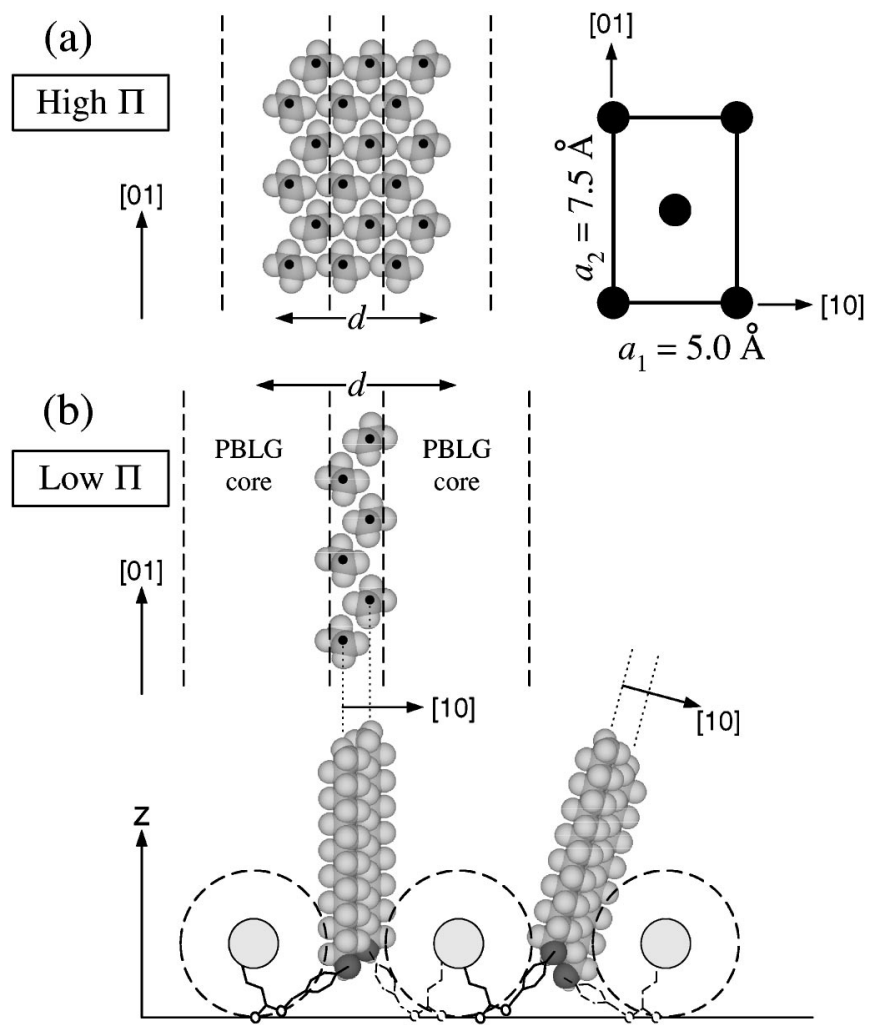

FIG. 4. A model for the HB packing of $-\mathrm{O}-\mathrm{C} 16$ chains, showing ordered chains only. (a) Top view at high $\Pi$. The [01] axis of the HB unit cell is parallel to the $\alpha$-helix axes of aligned PBLG cores. (b) Top and side views of zigzag packing at low $\Pi$ for - O- 16 chains that are confined between aligned PBLG cores. The chains can be untilted or tilted perpendicular to the core axes.

$\Pi$, the projection of $\xi_{11}$ onto the [10] axis (i.e., $\zeta_{10}$ $\left.=\xi_{11} a_{2} /\left[a_{1}^{2}+a_{2}^{2}\right]^{1 / 2}=0.83 \xi_{11}\right)$ reaches $\zeta_{10, \max }=11-14 \AA$ for PD $325(\Pi \gtrsim 25 \mathrm{dyn} / \mathrm{cm})$ and $\zeta_{10, \max }=17-21 \AA$ for MD76 ( $\Pi \gtrsim 15 \mathrm{dyn} / \mathrm{cm})$, which are comparable to the observed interhelix $d$ spacing, i.e., $\zeta_{10 \text {, max }} \sim d$. From this we infer that the local [01] axis runs parallel to the aligned PBLG cores, as shown in Fig. 4(a).

Third, the presence of a weak (02) peak and the near absence of the (11) peak for $\Pi \lesssim 5 \mathrm{dyn} / \mathrm{cm}$ indicate that although $-\mathrm{O}-\mathrm{C} 16$ chains are mostly disordered at low $\Pi$, a small fraction of them form a locally HB-packed structure. As shown in Fig. 2(b), the PBLG cores align locally without external pressure, implying strong mutual attraction. It follows that even at $\Pi=0$, the chains between aligned cores, which extend out to the vapor from below, are confined onedimensionally under a high local compressive stress. It would thus seem that the chain order appearing at low $\Pi$ [i.e., the $(02)$ peak] is a consequence of the $1 \mathrm{D}$ confinement between aligned PBLG cores. Moreover, the growth of the (11) peak with increasing $\Pi$ can be interpreted as enhancement of the HB order along the [10] axis perpendicular to the cores with decreasing interhelix $d$ spacing. On compression the unconfined chains sitting directly above the cores are brought into the HB structure. The conclusion that the HB order is first established along the helix backbone and then grows with increasing $\Pi$ is the most important message of this study. 
Figure 4(b) illustrates an idealized model of the initial HB structure. Two rows of $-\mathrm{O}-\mathrm{C} 16$ chains form a zigzag pattern in the confined region, where each row is contributed by one of the two neighboring C16-O-PBLG. The positions of chains along each row are periodic with repeat distance $a_{2}$ and are related to those of the other row by a vector $\left(a_{1} / 2, a_{2} / 2\right)$ in the plane normal to the chain axes. It is not possible to fit more than two rows within the gap $\Delta d \sim 5 \AA$ between aligned PBLG cores. As indicated in the figure, there is probably a distribution in the vertical tethering position of $-\mathrm{O}-\mathrm{C} 16$ chains within a given ordered domain, and for those domains that are tilted, the direction is transverse to the rod axis. Given $a_{2}=7.5 \AA=5 L_{1}$, the model implies that on average, every segment of five monomers in each rod would contribute one chain to the zigzag structure on one side of the core and another to the other side [32]. If this is true, the area taken up by a five-monomer segment should be approximately $A_{5} \sim 5 A_{\mathrm{PBLG}}+2 A_{\mathrm{HB}}$, where $A_{\mathrm{HB}}$ $=a_{1} a_{2} / 2=18.8 \AA^{2} /$ chain for the $\mathrm{HB}$ packing. Taking $A_{\mathrm{PBLG}} \sim 19.5 \AA^{2} /$ monomer for the core at low $\Pi$ [Fig. 1(a)], the model predicts the area/monomer for C16-O-PBLG to be $A_{5} / 5 \sim 27.0 \AA^{2} /$ monomer, which agrees well with $A=d$ $\times L_{1} \sim 28 \AA^{2} /$ monomer at $\Pi=0$ [Fig. 2(b)]. As for the unconfined chains above the core (roughly three per fivemonomer segment), the average area of $\frac{5}{3} A_{\mathrm{PBLG}}$ $\sim 32.5 \AA^{2} /$ chain available to each at low $\Pi$ is more than enough room for these chains to be disordered.

In summary, the system studied here is qualitatively different from any other system of alkyl chains studied to date in that 2D order grows out epitaxially from the 1D order of the confined chains. It should also be noted that according to the generalized phase diagram of fatty acid monolayers, the ordered phase formed by $\mathrm{C} 16$ acid at room temperature should have hexagonal symmetry and lower density than the HB-packed phases $L_{2}^{\prime \prime}$ (tilted to NN) and CS (untilted), which would only occur below $\sim-20^{\circ} \mathrm{C}$ [12]. The difference here is probably caused by confinement-induced reduction in the chain entropy. This is interesting because one should expect subtle changes in the properties of tethered chains as the chain length increases towards the polymer regime. For example, Kraack, Deutsch, and Sirota recently studied the effect of chain length variation on the degree of undercooling of $n$ alkanes [33]. We hope to continue the studies reported here with side chains of increasing length.

The Harvard contribution to this work was supported by Grant No. NSF-DMR-01-24936. D.T. acknowledges support by Grant No. NSF-DMR-01-10437. NSLS at Brookhaven National Laboratory is supported by No. DE-AC02$76 \mathrm{CH} 00016$.
[1] P. Flory, Principles of Polymer Chemistry (Cornell University Press, Ithaca, NY, 1953).

[2] P.-G. de Gennes, Scaling Concepts in Polymer Physics (Cornell University Press, Ithaca, NY, 1979).

[3] M. Muller and M. Schick, Phys. Rev. E 57, 6973 (1998).

[4] J. F. Nagle, Annu. Rev. Phys. Chem. 31, 157 (1980).

[5] J. F. Nagle, Faraday Discuss. Chem. Soc. 81, 151 (1986).

[6] G. L. Kenausis et al., J. Phys. Chem. B 104, 3298 (2000).

[7] D. H. Kim, A. L. Klibanov, and D. Needham, Langmuir 16, 2808 (2000).

[8] I. Szleifer and M. A. Carignano, Macromol. Rapid Commun. 21, 423 (2000).

[9] L. Leger, E. Raphael, and H. Hervet, Adv. Polym. Sci. 138, 185 (1999).

[10] S. Marcelja, Biochim. Biophys. Acta 367, 165 (1974).

[11] S. Marcelja, J. Chem. Phys. 60, 3599 (1974).

[12] V. M. Kaganer, H. Möhwald, and P. Dutta, Rev. Mod. Phys. 71, 779 (1999).

[13] I. Kuzmenko, V. M. Kaganer, and L. Leiserowitz, Langmuir 14, 3882 (1998).

[14] P.-G. de Gennes, Adv. Colloid Interface Sci. 27, 189 (1987).

[15] S. Alexander, J. Phys. (Paris) 38, 983 (1977).

[16] P. Lambooy et al., Phys. Rev. Lett. 72, 2899 (1994).

[17] D. M. Small, The Physical Chemistry of Lipids: From Alkanes to Phospholipids (Plenum Press, New York, 1986).

[18] O. M. Magnussen et al., Nature (London) 384, 250 (1996).

[19] L. Strong and G. M. Whitesides, Langmuir 4, 546 (1988).

[20] G. Y. Liu et al., J. Chem. Phys. 101, 4301 (1994).

[21] P. Fenter et al., Langmuir 7, 2013 (1991).

[22] I. M. Tidswell et al., J. Chem. Phys. 95, 2854 (1991).

[23] B. J. Factor et al., Phys. Rev. E 48, R2354 (1993).

[24] C. Prinz, P. Muller, and M. Maaloum, Macromolecules 33, 4896 (2000).

[25] S. M. Yu and D. A. Tirrell, Biomacromolecules 1, 310 (2000).

[26] D. K. Schwartz, M. L. Schlossman, and P. S. Pershan, J. Chem. Phys. 96, 2356 (1992).

[27] P. S. Pershan and J. Als-Nielsen, Phys. Rev. Lett. 52, 759 (1984).

[28] M. Fukuto et al., J. Chem. Phys. 111, 9761 (1999).

[29] M. Fukuto et al. (unpublished).

[30] B. M. Ocko et al., Phys. Rev. E 55, 3164 (1997).

[31] M. K. Durbin et al., Phys. Rev. E 58, 7686 (1998).

[32] We do not imply that every fifth monomer has its side chain protruding on the same side of the rod, which is inconsistent with the azimuthal angle change of $\Delta \phi=140^{\circ}$ around the helical axis over five monomers.

[33] H. Kraack, M. Deutsch, and E. B. Sirota, Macromolecules 33, 6174 (2000). 